\title{
TA KEO TEMPLE RECONSTRUCTION BASED ON TERRESTRIAL LASER SCANNING TECHNOLOGY
}

\author{
X. H. Xi ${ }^{\text {a b }}$, C. Wang ${ }^{\text {a, b* }}$, Y. P. Wan ${ }^{\text {a,b }}$, K. N. KHUON ${ }^{c}$ \\ ${ }^{a}$ Key Lab of Digital Earth, Institute of Remote Sensing and Digital Earth, Chinese Academy of Sciences, Beijing 100094, China; (xixh, \\ wangcheng,wanyp)@ radi.ac.cn \\ ${ }^{\mathrm{b}}$ International Centre on Space Technologies for Natural and Cultural Heritage under the Auspices of UNESCO, Beijing 100094, China \\ ${ }^{c}$ Authority for the Protection and Management of Angkor and the Region of Siem Reap, 999094, Cambodia
}

KEY WORDS: Terrestrial Laser Scanning Technology, 3D Reconstruction, Point Clouds, Registration, Digital Model, Ta keo Temple

\begin{abstract}
:
Ta Keo temple is one of the very famous temple complex of Angkor Wat in northwestern Cambodia. It has been suffering massive collapse and other serious damages in recent years. Nowadays, Terrestrial Laser Scanning(TLS) technology is considered as a wellestablished resource for heritage documentation and protection (Lerma et al, 2008; Reshetyuk, 2009). This paper used TLS to reconstruct Ta Keo Temple. Firstly, we acquired 71 scanning stations of points cloud data with high density and high accuracy, and over one thousand images with high spatial resolution about the temple. Secondly, the raw points cloud data were denoised, reduced and managed efficiently, and registrated using an adjusted ICP algorithm. Thirdly, a triangulation method was used to model most objects. At last, we mapped the texture data into the digital model and a 3-D model of Ta Keo with high accuracy was achieved. The authors focus on large object reconstruction by TLS technology, and pay much attention to the scanning design, multi-station data and the whole project's data registration, and texture mapping and so on. The research result will be useful for Ta Keo restoration, reconstruction and protection. Also, it is a good reference source for large complex buildings reconstruction when using terrestrial laser scanning technology.
\end{abstract}

\section{INTRODUCTION}

Angkor Wat is a medieval temple complex at Angkor in northwestern Cambodia and has been the focus of intensive scholarly research in recent decades of years (Evans et al, 2013). It was designated as a UNESCO world heritage site in 1992. However, it was listed as a danger heritage at the same year due to its massive collapse, weathering, flood and so on(Preston and Mccurry, 2000). So, there are many experts from many countries around the world to protect and try to restore it using modern methods during the past tens of years. However, well-known for their structural and surface complexity, the temples constitute a great challenge to any attempt to precise and detailed 3D measurements and modelling. Gruen et al (2005) deployed a pilot project using modern techniques of analytical and digital photogrammetry to derive a photorealistic 3D model of the famous Bayon temple in Angkor Thom. Meanwhile, a number of terrestrial images with a high resolution camera were taken. The ultimate goal of the project is to develop a system that is capable of producing high quality photorealistic 3D models. They generated a model with a mixture of manual (phototriangulation) and automated procedures (image matching for surface reconstruction, edit for blunder removal, texture mapping, visualization and animation) (Visnovcova et al, 2001). Remondino et al (2010) used aerial images to check the possibility to derive detailed and reality-based 3D models of complex historic buildings of the Wat. They generated ortho-photos by the method of image triangulation, geo-referencing, digital elevation model, and texturing commercial and in-housing programs. And in order to reconstruct the $3 \mathrm{D}$ model of the temples, manual stereo measurements were performed. However, the resolution of the available images was not sufficient for a detailed modelling of the study area.

Terrestrial Laser Scanning technology (TLS) is an enormous development in the techniques for spatial data acquisition. It employs an indirect ranging principle. The distance, or range from the sensor (ground laser scanner) to a point on the object surface is determined with high accuracy by measuring the time of flight between the emission of a laser signal and detection of its portion backscattered from the suface (Wehr and Lohr, 1999). By means of an opto-mechanical scanner, in both horizontal and vertical directions, the points cloud data of the whole object is achieved through scanning at the rates of thousands to hundreds of thousands of points per second depending on the ranging approach employed. The output of the procedure is a highly detailed 3D image of the object, typically consisting of billions of densely points. For each point, 3D coordinates in the coordinated system fixed to the scanner and the intensity of the reflected laser signal are recorded. Therefore, comparing with traditional surveying techniques, e.g. tacheometry, GPS and photogrammetry, TLS has numerous advantages, such as direct, raped and detailed capture of object geometry in 3D; dramatic reduction in costs and much faster project completion; possibility to survey remotely very complex, inaccessible and hazardous objects and areas, where the traditional techniques fail; no need for lighting for data acquisition; completeness and comprehensiveness of scanning, all the surfaces in the scene is captured at once(Reshetyuk, 2009). In this paper, the authors deploy terrestrial laser scanning technology in Ta Keo Temple, one the most important parts of Angkor Wat, in order to achieve a 3-D model of the temple.

\footnotetext{
* Corresponding author: Wang Cheng, wangcheng@ radi.ac.cn
} 


\section{MATERIALS AND DATA}

\subsection{Introduction to Ta Keo Temple}

Ta Keo temple is an approximate rectangle with 120 meters in length and 100 meters in width. Its main body was made with stones and bricks. The main axis of the temple is E-W and a causeway 500 meters long connects its eastern entrance to a landing stage on the Eastern Baray. The first terrace is $122 \mathrm{~m}$ by $106 \mathrm{~m}$, its wall of sandstone on laterite basis constitutes the outer enclosure(Freeman et al, 2006). It has five sanctuary towers arranged in a quincunx, built on the uppermost level of five-tier pyramid consisting of overlapping terraces. The central lotus tower is 20 meters high, see Fig. 1 and Fig.2. There are many collapsed walls everywhere.

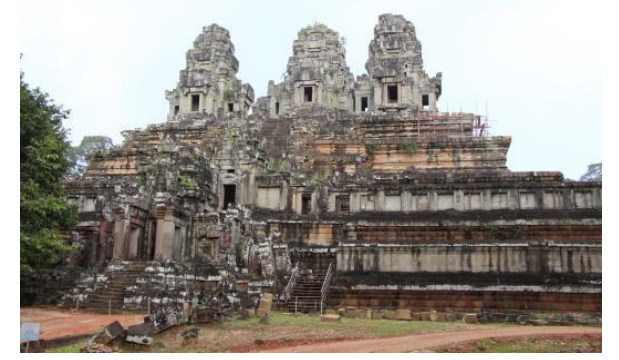

Fig.1 An overall of the Ta Keo Temple

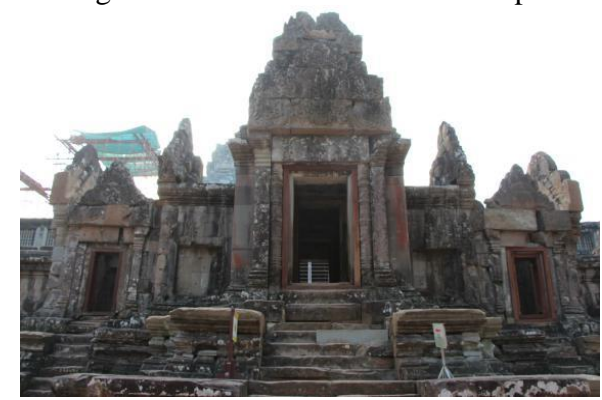

Fig. 2 The east gate of the Ta Keo Temple

\subsection{Data Acquisition}

Before data acquisition, we carried out several experiments under different weather conditions. The results show that there exists much noisy data in fog haze and rainy weather. Figure 3 and Figure 4 show the data results in these two conditions respectively using TLS. The blue points are noisy data. Although, TLS providers and users may think that TLS can work well in any weather conditions, we suggest it work in good weather in order to provide high quality data.

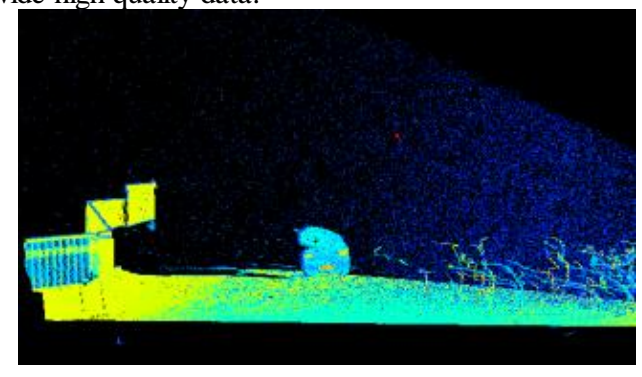

Fig. 3 Noisy data under fog haze weather condition

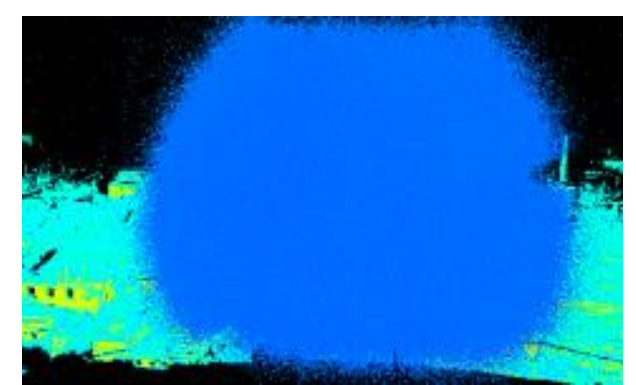

Fig.4 Noisy data under rainy weather condition Blue points are noise, which will affect data completion seriously. The equipment used in this study is Riegl VZ-1000. The scanner angle is from +60 degree to -40 degree in horizontal direction(Fig. 5). A platform is needed to get the complete data of the scanning area. According the temple characteristics, 70 scanning stations were arranged and points cloud data of 230GB and over one thousand images by the camera amounted on the scanner were obtained during the period of Nov, 1 to 4, 2013.

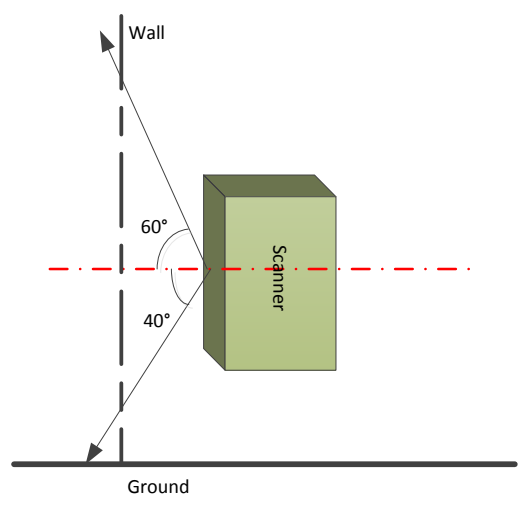

Fig. 5 Illustration of scanning empty area

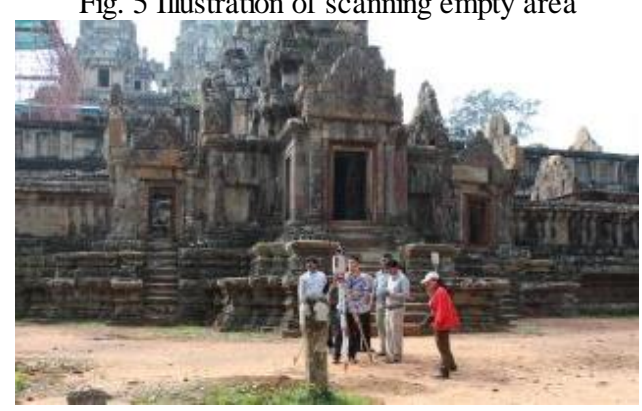

Fig.6 Field scanning photo in the east gate of Ta Keo Temple

\section{DATA REGISTRATION}

Two steps of registration were used in this study due to many scanning station and massive point clouds data. One is coarse registration, the other is precise registration.

\subsection{Coarse registration}

The first step in TLS data processing is registration of multiple scans and georeferencing. The latter procedure is transformation of the point clouds form the scanner coordinate system to a local or national coordinate system. It is very important for the integration of the TLS data and the products derived from it, e.g. 3D models and other geospatial data. Riscan Pro designed by the scanner hardware, provides several registration methods to combine multi-station points cloud data into on point cloud of the whole object or site. Then, the combined point cloud was 
transformed to the external coordinate system using the coordinates of minimum 3 well-distrbuted control points, realized by means of special targets placed on the nearby of the scanned object.

In this study, we use absolute registration method for neighbour stations data according to the GPS coordinates of the stations. Based on these results, every 5 stations data are registrated and errors are adjusted in order to depress the transmission errors.

\subsection{Precise registration}

Characteristic planes are produced using the point clouds data. And based on these planes, we used an adjusted ICP algorithm to achieve precise registration results. Using the minmum square sum of the distance difference of two planes as threshold value and a certain search radius, an iterative computation is produced which can shorten the calculation and reduce the error greatly.

Finally, all the stations data are integrated using regional adjustment and multi station adjustment methods.

Usually, there are much noise, error and redundant data in points cloud data which will affect the result of the 3-D digital model results. Most of noise and error can be deleted by filter method with man-machine interaction. As for the redundancy, a reduction method is used to filter it automatically. Figure 7shows the registration result of the part of the project.

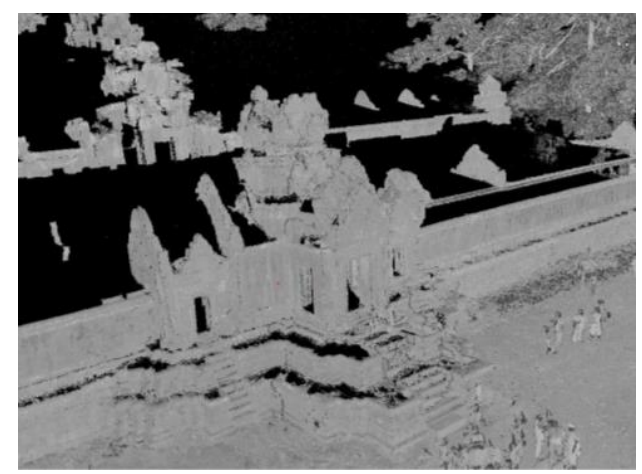

Fig.7 Registrated Points cloud of the east gate

\section{MODEL RECONSTRUCTION}

\subsection{Modeling}

With the high density points cloud data, we can build a 3-D digital model by using triangulation network method. Due to the large building and massive points cloud, the total data is segmented to many different parts. When each part is modeled, we integrate all of the models.

\subsection{Texture Data Mapping}

The true 3-D digital model is our final results. When we get the digital model, the texture data will be mapped into it by tiepoints in the model and its corresponding image. Figure 8 shows an example of a door frame model when mapping with texture data.

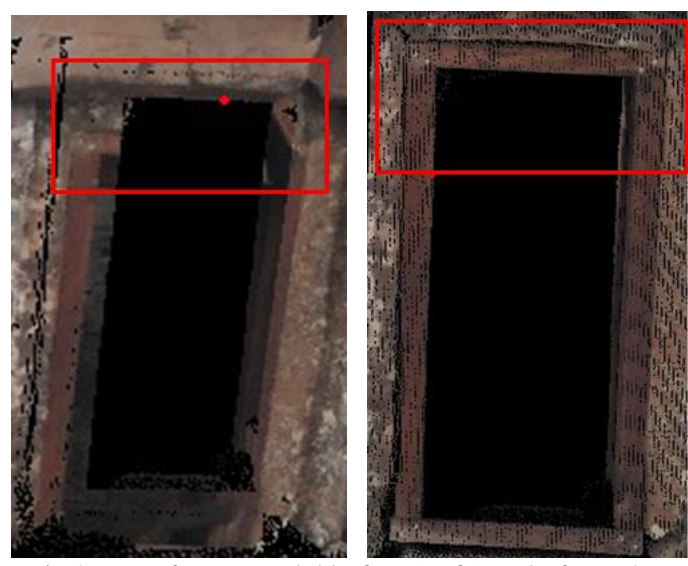

Fig. 8 Door frame model before(Left) and after when mapping the texture data(Right)

\subsection{3-D true model}

Figure 9 shows 3-D model of the east gate of Ta Keo.

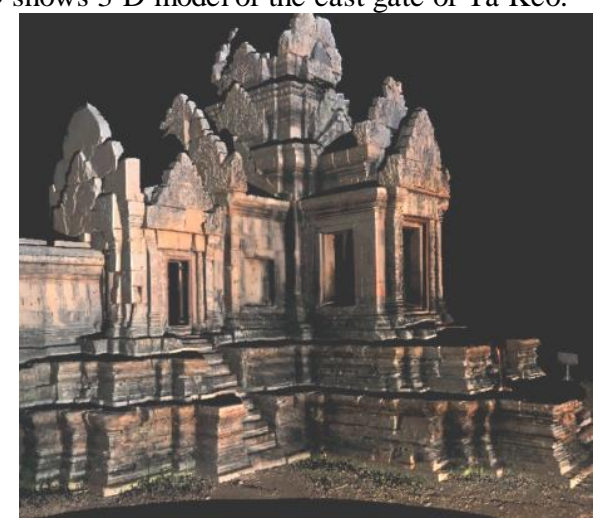

Fig.9 3-D model of the east gate of the Ta Keo temple

\section{CONCLUSIONS}

This paper explored a reconstruction method for large and complex object using terrestrial laser scanning technology including scanning design, data acquisition, preprocessing and modelling and so on. And for each step, we provide suitable scheme and proposal which is useful and a reference for other similar objects reconstruction when using TLS technology.

\section{ACKNOWLEDGEMENTS}

This work was supported by Bureau of International Cooperation, Chinese Academy of Sciences(241311KYSB20130001) and the Hundred Talents Program of the Chinese Academy of Sciences. The authors wants to thank many staffs from APSARA who assisted to acquire all of the data used in this paper.

\section{REFERENCES}

Evans D H, Fletchera R J, Pottier C, et al., 2013. Uncovering archaeological landscapes at Angkor using LiDAR. PNAS Early Edition, pp:1-6.

Freeman, Michael, Jacques, Claude, 2006. Ancient Angkor. River Books. ISBN: 974-8225-27-5. 
Gruen A, Remondino F, Zhang L., 2005. 3D Modeling and visualization of cultural heritage sites from high-resolution imagery. Proc. of the 25th Symposium of the European Association of Remote Sensing Laboratories (EARSeL), pp. 611-620.

Kersten T P, Lindstaedt M, Vogt B., 2009. Preserve the Past for the Future - Terrestrial Laser Scanning for the Documentation and Deformation Analysis of Easter Island's Moai. Photogrammetrie - Fernerkundung - Geoinformation, (1): 7990.

Lerma García J L, Van Genechten B, Santana Quintero M, 2008. 3D Risk Mapping. Theory and Practice on Terrestrial Laser Scanning. Training Material Based on Practical Applications. 2008.

Preston D, Mccurry, 2000. The Temples of Angkor-Still under attack. National Geographic Magazine, August 2000, pp: 84103.

Reshetyuk Y, 2009. Self-calibration and direct georeferencing in Terrestrial Laser Scanning. Sweden: Royal Institute of Technology.

Remondino F, Rizzi A, 2010. Reality-Based 3D Documentation Of Natural And Cultural Heritage Sites-Techniques, Problems, And Examples[J]. Applied Geomatics, 2(3): 85-100.

Visnovcova J, Zhang L, Gruen A., 2001. Generating a 3D model of a Bayon tower using non-metric imagery. Proceedings of the International Workshop Recreating the Past-Visualization and Animation of Cultural Heritage. Ayutthaya, Thailand, 26.2.-01.3, pp. $30-39$.

Wehr A, Lohr U, 1999. Airborne laser scanning - an introduction and overview. ISPRS Photogrammetry and Remote Sensing, 54: 68- 82. 